\title{
NATUREZA E CLASSIFICAÇÃO DAS INTERVENÇÕES DE ENFERMAGEM EM AMBULATÓRIO DE QUIMIOTERAPIA DE ADULTOS*
}

\author{
Maria Gaby Rivero de Gutiérrez** \\ Nilce Piva Adami*** \\ Rosa Apparecida Pimenta de Castro**** \\ Selma Montosa da Fonseca*****
}

GUTIÉRREZ, M.G.R.; ADAMI, N.P.; CASTRO, R.A.P.; FONSECA, S.M. Natureza e classificação das intervenções de enfermagem em ambulatório de quimioterapia de adultos. Rev.latino-am.enfermagem, Ribeirão Preto, v. 8, n. 3, p. 33-39, julho 2000 .

Este estudo descritivo objetivou identificar, por meio da análise retrospectiva de registro, a natureza das intervenções de enfermagem e estabelecer a correspondência entre estas e os problemas levantados. A população abrangeu 184 prontuários de pacientes atendidos na primeira consulta de enfermagem no Ambulatório de Quimioterapia de Adultos do Hospital São Paulo, no periodo de janeiro a junho de 1997, selecionando-se uma amostra aleatória de 37 prontuários, sendo 19 de pacientes do sexo masculino e 18 do feminino. Os principais resultados mostraram o predomínio das intervenções centradas no atendimento das necessidades psicobiológicas, sendo que das psicossociais, as intervenções educativas foram as mais freqüentes. A correspondência entre problemas identificados e intervenções propostas, foi mais evidente no atendimento das necessidades psicobiológicas.

UNITERMOS: assistência ambulatorial, quimioterapia, enfermagem oncológica, avaliação de processos, cuidados de saúde

\section{INTRODUÇÃO}

O Hospital São Paulo da Universidade Federal de São Paulo, faz parte da rede de serviços de saúde do Núcleo Regional de Saúde 5 da Secretaria de Estado da Saúde de São Paulo, sendo um centro de referência de nível terciário para a população residente na área abrangida por esse Núcleo.

Esta instituição constitui também, um dos centros de referência do Município de São Paulo para o tratamento de câncer. Nesse serviço tem sido registrado um aumento crescente do número de atendimentos, ambulatorial e de internação, de pacientes com neoplasias malignas, dos quais cerca de $70 \%$ em estágios avançados da doença e que, em 1997, representou a segunda causa de óbito nesse hospital.

O incremento dessa demanda pode ser explicado pela transição demográfico-epidemiológica observada em nosso país que produziu o envelhecimento da população, e alterou significativamente o perfil de morbi-mortalidade da população brasileira.
No que se refere às neoplasias malignas no Brasil, a industrialização, a urbanização, a exposição freqüente a uma gama de produtos potencialmente cancerígenos e a expectativa maior de vida contribuem para que o câncer venha assumindo uma importância relativa cada vez maior entre as causas de morte. De 1930 para 1989, as neoplasias aumentaram como causa dos óbitos ocorridos (3\% a $12 \%$, respectivamente), representando atualmente a segunda causa de morte por doença no país (BRASIL, 1996).

A estimativa de mortalidade para 1998 prevê a ocorrência de 107.950 óbitos por câncer, sendo 58.070 $(53,8 \%)$ no sexo masculino e $49.880(46,2 \%)$ no sexo feminino. Os tumores responsáveis pelas mais altas taxas de incidência e mortalidade no país são: pulmão, estômago e próstata para o sexo masculino e mama, cérvico-uterino e estômago para o sexo feminino. Foi estimado, também, que ocorrerão 269.000 casos novos de câncer, sendo 140.705 (52,3\%) em mulheres e 128.295 $(47,7 \%)$ em homens. Dos casos novos em mulheres, 32.695 (12,1\%) corresponderão ao câncer de mama e,

\footnotetext{
* Trabalho apresentado no VI Colóquio Pan-Americano de Investigação em Enfermagem. Ribeirão Preto, 19 a 25 de maio de 1998

** Enfermeira. Doutor em Enfermagem e Professor Adjunto do Departamento de Enfermagem da UNIFESP/EPM

*** Enfermeira. Professor Titular do Departamento de Enfermagem da UNIFESP/EPM

**** Enfermeira. Professor Adjunto do Departamento de Enfermagem da UNIFESP/EPM

***** Enfermeira do Ambulatório de Quimioterapia de Adultos do HSP, Especialista em Enfermagem Oncológica e Mestranda em Enfermagem da Escola de Enfermagem da Universidade de São Paulo
} 
$21.725(8,0 \%)$ ao câncer de colo uterino. Nos homens é previsto que ocorrerão $15.040(20,7 \%)$ casos novos de câncer de pulmão e 14.665 (20,2\%) casos de câncer de próstata (BRASIL, 1998).

Este quadro também se reproduz no Estado de São Paulo, sendo que a maior diferença é em relação ao câncer de colo uterino que ocupa o $5^{\circ}$ lugar (FONSECA, 1995).

A magnitude dos dados acima apresentados, torna relevante a necessidade da atuação do Núcleo de Enfermagem em Oncologia (NEO) do Departamento de Enfermagem da UNIFESP/EPM, na participação dos esforços para o atendimento efetivo e de melhor qualidade prestado à clientela portadora de câncer atendida no HSP.

Dentre os vários projetos desenvolvidos pelo NEO destaca-se o centrado na melhoria do atendimento ambulatorial aos pacientes submetidos à quimioterapia antineoplásica. Para tanto, membros deste Núcleo e alunos de graduação em enfermagem desenvolveram estudos, um dos quais, voltado para a elaboração de um instrumento de coleta de dados para avaliação diagnóstica dos problemas de enfermagem apresentados por esses pacientes (GUTIÉRREZ et al., 1995).

O instrumento derivado desse trabalho tem sido utilizado na consulta de enfermagem prestada aos pacientes atendidos no Ambulatório de Quimioterapia de Adultos do HSP.

Esta atividade, determinada na legislação brasileira sobre o exercício profissional de enfermagem como privativa do enfermeiro, foi implantada nessa unidade em janeiro de 1997, com vistas a sistematizar o processo assistencial de enfermagem, possibilitando, ainda, melhor preparo de alunos de enfermagem, bem como a realização de pesquisas sobre a qualidade dos cuidados prestados. Esta sistematização faz parte da filosofia da Diretoria de Enfermagem do hospital e está sendo implantada, também, nas unidades de internação objetivando alcançar o patamar desejável para a assistência de enfermagem.

Visando melhorar a implementação desta atividade, FONSECA et al. (1997) realizaram uma pesquisa que mostrou a necessidade de reformular a consulta de enfermagem, tendo em vista a identificação dos problemas do paciente na sua integralidade, bem como a explicitação dos diagnósticos e intervenções de enfermagem, de modo a possibilitar a avaliação do alcance dos resultados das ações implementadas.

A constatação dessa situação motivou as autoras a dar continuidade às pesquisas avaliativas a fim de conhecer melhor quais as intervenções específicas de enfermagem implementadas pelas enfermeiras do Ambulatório supracitado com vistas a prevenir, aliviar ou resolver os problemas por elas identificados.

Para orientar o desenvolvimento deste estudo, no referente à avaliação da qualidade, foi adotada a abordagem de Processo proposta por DONABEDIAN (1993) enfocando primordialmente a coerência existente entre problemas levantados e intervenções prescritas ao paciente em tratamento quimioterápico.

Para a identificação das necessidades destes pacientes, utilizou-se o modelo teórico de HORTA (1979) e, para analisar as intervenções prescritas, a Classificação de Intervenção de Enfermagem (Mc CLOSKEY \& BULECHEK, 1996).

Assim sendo, os objetivos estabelecidos para este estudo foram:

- Identificar a natureza das intervenções de enfermagem; e,

- Analisar a correspondência existente entre estas e os problemas levantados.

\section{METODOLOGIA}

Trata-se de um estudo descritivo, retrospectivo, realizado no Ambulatório de Quimioterapia de Adultos do Hospital São Paulo, hospital de ensino de porte extra (614 leitos ativos) que presta assistência de nível terciário.

A população de estudo abrangeu 184 prontuários de pacientes submetidos à quimioterapia antineoplásica, no período de janeiro a junho de 1997 e que foram atendidos na consulta de enfermagem pela primeira vez, dos quais foi selecionada uma amostra aleatória sistemática de 37 prontuários (20,1\%), sendo 19 de pacientes do sexo masculino e 18 do sexo feminino.

Para estabelecer o número amostral considerouse que $20 \%$ seria suficiente, uma vez que no processo de auditoria de prontuários de pacientes hospitalizados é estabelecido um padrão internacional de 10\% (PEREIRA \& TAKAHASHI, 1991).

Para a coleta de dados foi utilizado um instrumento contendo itens relativos a: sexo, escolaridade, idade, diagnóstico médico, problemas e intervenções de enfermagem, que tem sido fonte de informações para as pesquisas que vem sendo realizadas nesse Ambulatório. Neste estudo foram selecionados os dados referentes a problemas e intervenções de enfermagem conforme apontados na Figura 1.

Os problemas identificados foram categorizados conforme proposta de HORTA (1979) em: problemas relacionados às necessidades humanas básicas de ordem psicobiológica, psicossocial e psicoespiritual. As intervenções prescritas foram agrupadas de acordo com a Nursing Interventions Classification - NIC (Mc CLOSKEY \& BULECHEK, 1996), uma vez que o referencial de Horta utilizado para classificar os problemas identificados, não contêm os subsídios necessários ao propósito deste estudo.

Precedendo a realização desta investigação, encaminhou-se o Projeto de Pesquisa ao Comitê de Ética em Pesquisa desse hospital, obtendo-se a aprovação do mesmo. 


\section{RESULTADOS E DISCUSSÃO}

Tendo em vista elucidar o agrupamento efetuado, apresenta-se na Figura 1 os dados constantes no impresso da consulta de enfermagem prestada a um paciente em tratamento quimioterápico, conforme o registro realizado pela enfermeira.

\begin{tabular}{|c|c|c|c|}
\hline Paciente & Problemas identificados & Informações comple mentares & Intervenções prescritas \\
\hline M.Q.M. & $\begin{array}{l}\text { - Sonolência } \\
\text { - Fadiga } \\
\text { - Tristeza } \\
\text { - Ansiedade } \\
\text {-Diminuição da auto estima } \\
\text { - Náusea } \\
\text { - Alopécia } \\
\text { - Tontura } \\
\text { - Não se relaciona sexualmente } \\
\text { com o marido } \\
\text { - Separou roupas e utensilios por } \\
\text { acreditar que a doença é } \\
\text { contagiosa }\end{array}$ & $\begin{array}{l}\text { Paciente do sexo feminino, } 45 \\
\text { anos, } 1^{\circ} \text { grau incompleto, com } \\
\text { diagnóstico médico de Neo de } \\
\text { mama, recebendo o esquema } \\
\text { quimioterápico: } \\
\text { - Methotrexate } 67 \mathrm{mg}\} \\
\text { - Fluorouracil } 100 \mathrm{mg} \text { \} EV* } \\
\text { - Ciclofo sfamida } 100 \mathrm{mg} \text { \} } \\
* 1 \text { aplicação a cada } 28 \text { dias }\end{array}$ & $\begin{array}{l}\text { Orientar quanto: } \\
\text { - tratamento } \\
\text { - efeitos colaterais da } \\
\text { quimioterapia } \\
\text { - doença não ser transmissive } \\
\text { - possibilidade de } \\
\text { relacionamento sexual com o } \\
\text { marido } \\
\text { - evitar exposição ao sol }\end{array}$ \\
\hline
\end{tabular}

Figura 1 - Súmula dos dados extraídos do registro de uma consulta de enfermagem efetuada em ambulatório de Quimioterapia de Adultos. São Paulo, 1997

A seguir, expõe-se o agrupamento das intervenções de enfermagem, segundo a sua natureza e tipo de ação realizada.

Tabela 1 - Natureza e tipo de intervenções identificados na consulta de enfermagem em Ambulatório de Quimioterapia de Adultos. São Paulo, 1997

\begin{tabular}{lll}
\hline \multicolumn{1}{c}{ Natureza } & \multicolumn{1}{c}{ Tipo } & $\mathrm{n}^{\circ}$ \\
\hline Fisiológica & & \\
- Cuidados que dãosuporte & - Suporte nutricional & 32 \\
ao funcionamento fisico & - Facilitação do auto-cuidado & 07 \\
& -Monitoração das eliminaços & 04 \\
& - Promoção do conforto fisico & 02
\end{tabular}

Sub Total

45

Comportamental

- Cuidaddos que dão suporte ao funcionamento psicossocial e facilitam as mudarças no estilo de vida

\section{Sub Total}

Familiar

- Cuidados que dãosuporte - Cuidado na gestação e parto

à unidade farniliar

\section{Sub Total}

\section{Sistemade Saúde}

-Cuidados que dãosuporte - Intermediação com ao uso efetwo do sistema profissionais do sistema de de prestação de serviços à saúde saúde

\section{Sub Total}

Pelos dados da Tabela 1, pode-se constatar que as intervenções de natureza fisiológica e comportamental constituíram a base das prescrições de enfermagem (45 e 40 respectivamente).

O predomínio de prescrições direcionadas ao suporte nutricional e educação do paciente, justifica-se pela necessidade de orientar o paciente e familiares a controlar ou minorar os efeitos adversos decorrentes do tratamento quimioterápico, entre os quais destacam-se as náuseas, vômitos, mal estar e anorexia (GATTAES, 1996).

Cabe ressaltar que a prescrição de intervenções de natureza comportamental aponta para o avanço na direção de um atendimento mais abrangente do paciente, rompendo assim, um tipo de ação fortemente centrada nos aspectos fisiopatológicos.

Quanto às ações de intermediação das enfermeiras com profissionais do sistema de saúde, tem se destacado aquelas relacionadas a assegurar a oferta de medicamentos específicos e coadjuvantes requeridos ao tratamento do paciente com câncer. Esta situação decorre, por um lado, da precariedade de recursos financeiros repassados ao Sistema Único de Saúde e por outro, pelo afastamento de pacientes do mercado de trabalho reduzindo seu poder aquisitivo e de compra de medicamentos.

Buscando responder à questão básica que conduziu este estudo, ou seja, se o conhecimento dos problemas dos pacientes estaria norteando as intervenções de enfermagem, procedeu-se ao relacionamento destes conforme apresentado nas Tabelas 2 e 3 . 
Tabela 2 - Correspondência entre problemas identificados e intervenções prescritas na área psicobiológica, na consulta de enfermagem em Ambulatório de Quimioterapia de Adultos. São Paulo, 1997

\begin{tabular}{|c|c|c|c|c|c|}
\hline $\begin{array}{l}\text { Necessidades* } \\
\text { Psicobiológicas }\end{array}$ & Problemas * & & Interve nções & Tipo de ação & \\
\hline - Nutrição/hidratação & $\begin{array}{l}\text { Náusea } \\
\text { Anorexia } \\
\text { Azia } \\
\text { Desidratação }\end{array}$ & $\begin{array}{l}26 \\
05 \\
01 \\
01\end{array}$ & $\begin{array}{l}\text { Suporte Nutricional } \\
\text { (aconselhamento nutricional) }\end{array}$ & $\begin{array}{l}\text { Aumento da ingesta hídrica } \\
\text { Fracionar a dieta } \\
\text { Aumento da ingesta alimentar } \\
\text { Preparo de alimentos } \\
\text { Alimentação e hidratação }\end{array}$ & $\begin{array}{l}13 \\
12 \\
05 \\
01 \\
01\end{array}$ \\
\hline Sub Total & & $\mathbf{3 3}$ & & & 32 \\
\hline - Oxigenação & $\begin{array}{l}\text { Sonolência } \\
\text { Fraqueza/cansaço } \\
\text { Dispnéia } \\
\text { Tontura }\end{array}$ & $\begin{array}{l}12 \\
05 \\
03 \\
02\end{array}$ & $\begin{array}{l}\text { Promoção do conforto fisico } \\
\text { (Controle do ambiente) }\end{array}$ & Repouso após $\mathrm{QT}$ & 02 \\
\hline Sub Total & & 22 & & & 02 \\
\hline - Eliminação Intestinal & $\begin{array}{l}\text { Diarréia } \\
\text { Obstipaçãa }\end{array}$ & $\begin{array}{l}10 \\
03\end{array}$ & $\begin{array}{l}\text { Suporte Nutricional } \\
\text { (aconselhamento nutricional) }\end{array}$ & $\begin{array}{l}\text { Dieta obstipante } \\
\text { Dieta laxativa } \mathrm{p} / \text { diabético }\end{array}$ & $\begin{array}{l}02 \\
01\end{array}$ \\
\hline Sub Total & & 13 & & & $\mathbf{0 3}$ \\
\hline - Eliminação urinária & $\begin{array}{l}\text { Disúria } \\
\text { Incontinência } \\
\text { Hematúria }\end{array}$ & $\begin{array}{l}04 \\
01 \\
01\end{array}$ & $\begin{array}{l}\text { Facilitação do autocuidado } \\
\text { (cuidados perineal e na } \\
\text { incontinência urinária) }\end{array}$ & $\begin{array}{l}\text { Higiene corporale uso adequado } \\
\text { de fralda }\end{array}$ & 01 \\
\hline Sub Total & & 06 & & & 01 \\
\hline $\begin{array}{l}\text { - Integridade cutâneol } \\
\text { mucosa }\end{array}$ & $\begin{array}{l}\text { Colostomia } \\
\text { definitiva }\end{array}$ & 01 & $\begin{array}{l}\text { Monitoração da eliminação } \\
\text { (cuidado com a ostomia) }\end{array}$ & $\begin{array}{l}\text { Orientada quarto aos cuidados } \\
\text { com a colostomia }\end{array}$ & 01 \\
\hline Sub Total & & 01 & & & 01 \\
\hline TOTAL & & 75 & & & 39 \\
\hline
\end{tabular}

* Foram identificados problemas, sem registro de intervenções, referentes às necessidades de: integridade física (22), regulação neurológica (6), percepção dolorosa (4), exercício e atividade física (2), sono e repouso (1) e regulação hidrossalina (1)

Pelos dados da Tabela 2 pode-se verificar que foram identificados 75 problemas relacionados às necessidades psicobiológicas para os quais foram prescritas 39 intervenções de enfermagem, predominando as ações direcionadas ao aconselhamento nutricional (32).

Analisando o conteúdo das prescrições, observou-se que o registro das ações apresenta-se de modo genérico, uma vez que não constam informações sobre quem, o quê, onde, quando, como e com que freqüência deverão ser realizadas as atividades específicas para prevenção, alívio ou solução dos problemas identificados.

Outro aspecto observado foi a não explicitação dos resultados esperados a partir das ações implementadas. A falta de parâmetros para a mensuração dos resultados alcançados, compromete a avaliação da efetividade das intervenções realizadas. Pode-se inferir que esta situação decorra de comportamentos já incorporados na prática de enfermagem e da falta de ações de educação continuada. No entanto, a formalização da consulta de enfermagem há dois anos e a disponibilidade de bibliografia básica em língua portuguesa sobre cuidados de enfermagem em quimioterapia (BONASSA, 1992; AHANA \& KUNISHI, 1992; FUNDAÇÃO ONCOCENTRO, s.d.), não foram suficientes para operar as mudanças necessárias no processo assistencial de enfermagem. Acredita-se que mudanças dessa ordem, requerem intervenções nos âmbitos técnico, político e organizacional.

Chamou atenção ainda, a ausência de registros de intervenções para 36 problemas identificados na área psicobiológica, pois, supostamente, esta é uma das áreas 
de maior domínio dos profissionais de enfermagem. Segundo as enfermeiras do setor, muitas das ações implementadas não são registradas por fazerem parte do repertório habitual das orientações por elas ministradas durante a consulta de enfermagem. Também foi referido que algumas questões de âmbito pessoal do paciente, ficam restritas ao momento da interação enfermeira/ paciente, sem registro posterior das orientações dadas, quer por entenderem que esse é o desejo do paciente, quer por solicitação explícita do mesmo. Sabe-se que o cuidado do paciente é permeado de situações que envolvem aspectos éticos e as enfermeiras devem ter a sensibilidade e o conhecimento necessários para lidar com essas questões.

No entanto, acredita-se que a força do hábito de cuidar de forma assistemática gera lacunas ou mesmo imperfeições em todo o processo assistencial, inclusive na documentação e registro da assistência, o que dificulta a realização de estudos avaliativos.

No que tange à correspondência entre problemas identificados e prescrições de enfermagem, ainda que genéricas e incompletas, pode-se dizer que houve certa adequação entre ambos, salvo às referentes aos problemas relativos às necessidades de oxigenação e eliminação urinária.

Tabela 3 - Correspondência entre problemas identificados e intervenções prescritas na área psicossocial, na consulta de enfermagem em Ambulatório de Quimioterapia de Adultos. São Paulo, 1997

\begin{tabular}{|c|c|c|c|c|c|}
\hline $\begin{array}{l}\text { Necessidades } \\
\text { Psicossociais }\end{array}$ & Problemas & & Interve nções & Tipo de ação & \\
\hline $\begin{array}{l}\text { - Aprendizagem } \\
\text { (educaçãoà saúde) }\end{array}$ & $\begin{array}{l}\text { desconhece o tratamento } \\
\text { desconhece a doença } \\
\text { tem medo de contagiar o } \\
\text { marido }\end{array}$ & $\begin{array}{l}07 \\
01 \\
01\end{array}$ & $\begin{array}{l}\text { Educação do paciente (educação } \\
\text { em saúde, ensino sobre o } \\
\text { processo da doença e plano } \\
\text { terapêutico) }\end{array}$ & $\begin{array}{l}\text { Orientado quanto à: } \\
\text { efeitos colaterais da QT } \\
\text { doença e o tratamento } \\
\text { QT } \\
\text { doença } \\
\text { importância do autocuidado }\end{array}$ & $\begin{array}{l}18 \\
04 \\
09 \\
03 \\
01\end{array}$ \\
\hline Sub Total & & 09 & & & 35 \\
\hline $\begin{array}{l}\text { - Auto-estima e } \\
\text { auto-imagem }\end{array}$ & $\begin{array}{l}\text { perda da mama } \\
\text { vergonha dos amigos } \\
\text { presença de colostomia }\end{array}$ & $\begin{array}{l}02 \\
01 \\
01\end{array}$ & $\begin{array}{l}\text { Assistênciapara o enfrentamento } \\
\text { (estimular o enfrentamento) }\end{array}$ & $\begin{array}{l}\text { Procurar grupos de auto- } \\
\text { ajuda }\end{array}$ & 01 \\
\hline Sub Total & & 04 & & & 01 \\
\hline $\begin{array}{l}\text { - Aceitaçãol } \\
\text { segurança }\end{array}$ & $\begin{array}{l}\text { ansiedade, angústia, } \\
\text { nervosismo } \\
\text { depressão e tristeza } \\
\text { revolta, não aceita a } \\
\text { doença }\end{array}$ & $\begin{array}{l}12 \\
10 \\
01\end{array}$ & $\begin{array}{l}\text { Assistênciapara o enfrentamento } \\
\text { (suporte emocional estímulopara } \\
\text { o enfrentamento) }\end{array}$ & $\begin{array}{l}\text { Oferecer apoio emocional para } \\
\text { amenizar a ansiedade } \\
\text { Encaminhada para } \\
\text { psicológico }\end{array}$ & $\begin{array}{l}01 \\
01\end{array}$ \\
\hline
\end{tabular}

Sub Total

23

02

- Segurança emocional

$\begin{array}{lll}\text { medo dos efeitos daQT } & 01 & \text { Assistênciapara o enfrentamento } \\ \text { rejeição pelo marido } & 01 & \text { (suporte para tomada de decisão) } \\ \text { perda da mama } & 01 & \\ \text { medo do futuro } & 01 & \\ \text { medo indefinido } & 01 & \\ \text { medo com o resultado } & 01 & \\ \text { do tratamento } & \end{array}$

Orientação quanto à:

efeitos da QT 01

cirurgia e tipo de anestesia possibilidade de continuar trabalhando, e poupar-se na 01 medida do possivel

\begin{tabular}{lll}
\hline Sub Total & 06 & 03 \\
\hline TOTAL & 42 & 41 \\
\hline
\end{tabular}

Os dados apresentados na Tabela 3, demonstram que as ações voltadas para à educação do paciente foram as que tiveram maior destaque no conjunto de intervenções na área psicossocial (35), bem como correspondência com os problemas identificados nesta área. Observa-se, no entanto, que estas ações estão 
direcionadas ao ensino sobre o processo da doença e conseqüentemente, ao plano terapêutico. A ênfase dada à orientação sobre os efeitos colaterais da quimioterapia pode ser explicada pela crença de que o paciente informado poderá se beneficiar por meio da utilização de medidas que venham a atenuar esses efeitos, principalmente, no que se refere ao desconforto físico e emocional.

Quanto aos problemas relacionados às necessidades de auto-estima, auto-imagem, aceitação e segurança emocional, as intervenções de enfermagem ainda são escassas, não cobrindo, portanto, toda a gama desses problemas. Esta situação é freqüentemente justificada pela falta de instrumentalização básica na graduação em Enfermagem, tanto para abordagem do paciente como para intervir em situações onde estes aspectos estejam presentes.

Se por um lado esta justificativa pode estar gerando esta lacuna no atendimento, por outro, a falta de uma equipe multidisciplinar no Ambulatório de Quimioterapia de Adultos que possa dar suporte às dimensões psicossociais e psicoespirituais envolvidas no processo de adoecer, torna a situação mais difícil. Considera-se que a presença de outros profissionais, além do médico e da enfermeira é indispensável para propiciar uma assistência integral e de melhor qualidade aos pacientes com câncer.

\section{CONCLUSÕES}

Os principais resultados demonstraram que houve predomínio das intervenções centradas no atendimento das necessidades psicobiológicas, com destaque para a terapêutica, face ao uso de antineoplásicos que produzem efeitos colaterais esperados.

Quanto às psicossociais as intervenções de natureza educativa, visando a informação do paciente sobre o processo da doença e do tratamento e sua participação no auto-cuidado foram as mais freqüentes.

A correspondência entre problemas identificados e intervenções propostas foi mais evidente no atendimento das necessidades psicobiológicas, havendo, ainda, ausência de registro de intervenções para alguns problemas identificados, tanto nesta área, quanto na psicossocial.

Constatou-se também, que no planejamento das intervenções de enfermagem, não houve formulação de objetivos ou metas a serem alcançadas, que constituam parâmetros essenciais para avaliar os resultados da assistência prestada.

\section{CONSIDERAÇÕES FINAIS}

A realização deste estudo mostrou a importância da continuidade de pesquisas avaliativas sobre as propostas de mudança no processo assistencial de enfermagem que estão sendo implementadas no Ambulatório de Quimioterapia de Adultos do HSP.

Acredita-se que a participação das enfermeiras do setor e de docentes de enfermagem num grupo de pesquisa relacionado à Oncologia tem-se constituído em um fator positivo na elaboração de projetos que visam a melhoria da assistência e do ensino de enfermagem nessa área. Os resultados práticos já estão se fazendo sentir, na medida em que um número crescente de alunos de graduação procura o NEO para participar das atividades de iniciação científica e as três enfermeiras responsáveis pelo Ambulatório estão cursando programas de Mestrado.

Outro resultado prático, decorrente dos estudos realizados, inclusive deste, foi a elaboração de um Manual para orientar a execução de procedimentos de atendimento no Ambulatório de Quimioterapia de Adultos.

No entanto, diante das lacunas identificadas na implementação da consulta de enfermagem, fica evidente a necessidade de melhor preparo dos executores desta atividade e da ação efetiva de uma equipe interdisciplinar com vistas a produzir melhoria nos cuidados prestados ao paciente com câncer.

Ao lado destas medidas é indispensável também, a elaboração de protocolos específicos sobre os cuidados de enfermagem a serem prestados ao paciente em quimioterapia e o aperfeiçoamento das anotações de enfermagem no prontuário do paciente, uma vez que estes registros constituem uma das fontes de dados para a avaliação da qualidade da assistência prestada.

\section{NATURE AND CLASSIFICATION OF NURSING INTERVENTIONS IN AN ADULT CHEMOTHERAPY OUTPATIENT UNIT}

The purpose of the present descriptive study was to identify, through a retrospective analysis of records, the nature of nursing interventions as well as to establish the relationship between the nature of these interventions and the problems found. The sample was formed by 184 records of patients in their first nursing consultation at the Adult Chemotherapy Outopatiet Unit of Hospital São Paulo from January to June,1997; 37 records were selected at random: 19 male and 18 female patients. Results showed the predominance of interventions centered in patients' psychobiological needs. Educative interventions were more 
frequently performed when related to patients psychosocial needs. The correspondence between the identified problems and proposed interventions was evidenced when the psychobiological needs were met.

KEY WORDS: outpatient care unit, chemotherapy, oncology nursing, process assessment, health care

\section{NATURALEZA Y CLASIFICACIÓN DE LAS INTERVENCIONES DE ENFERMERÍA EN UN AMBULATÓRIO DE QUIMIOTERAPIA DE ADULTOS}

Este estudio retrospectivo tuvo como objetivos identificar la naturaleza de las intervenciones de enfermería y establecer la correspondencia entre estas y los problemas de los pacientes. De un total de 184 expedientes clínicos de pacientes atendidos en la primera consulta de enfermería en el ambulatorio de Quimioterapia de Adultos del Hospital São Paulo, en el periodo de enero a junio de 1997, fue seleccionada aleatoriamente una muestra de 37 expedientes clínicos, siendo 19 de pacientes del sexo masculino y 18 del femenino. Los principales resultados muestran el predominio de las intervenciones dirigidas para la atención de necesidades de orden psico-biológica, habiendo, consecuentemente, una mayor correspondencia entre estas y las intervenciones prescritas. En relación con las psico-sociales, las intervenciones relacionadas con el aprendizaje del paciente fueron las más frecuentes.

TÉRMINOS CLAVES: atención ambulatoria, quimioterapia, enfermería oncológica, evaluación de procesos, cuidados en salud

\section{REFERÊNCIAS BIBLIOGRÁFICAS}

01. AhanA, D.N.; KUniShi, M. Protocolos de enfermagem para o tratamento de paciente oncológico. 2. ed. São Paulo: Organização Andrei Editora, 1992.

02.BONASSA, E.M.A. Enfermagem em quimioterapia. São Paulo: Atheneu, 1992.

03. BRASIL. Ministério da Saúde. Secretaria Nacional de Assistência à Saúde. Instituto Nacional de Câncer. Coordenação da Programas de Controle de Câncer-Pro-Onco. Estimativa da incidência e mortalidade por câncer no Brasil, 1996. Rio de Janeiro: Pro-Onco/Inca, 1996.

04. BRASIL. Ministério da Saúde. Secretaria Nacional de Assistência à Saúde. Instituto Nacional de Câncer. Coordenação de Programas de Controle de Câncer - Pro-Onco. Estimativa da incidência e mortalidade por câncer no Brasil, 1998. Rio de Janeiro: Pro-Onco/INCA, 1998.

05. DONABEDIAN, A. Evaluación de la calidad de la atención médica. In: WHITE, K.L. (coord.). Investigación sobre servicios de salud: una antologia. Washington, D.C.: Organización Panamericana de Salud, 1993 (OPAS - Publ. Cient. 534).

06. FONSECA, L.A.M. A evolução das doenças neoplásicas. In: MONTEIRO, C.A.(org.). Velhos e novos males da saúde no Brasil. São Paulo: HUCITEC-NUPES/USP, 1995. p. 268-78.
07. FONSECA, S.M. et al. A consulta de Enfermagem em ambulatório de quimioterapia de adultos. In: CONGRESSO BRASILEIRO DE ENFERMAGEM, 49. Belo Horizonte, 1997. Programa. Belo Horizonte: ABEn-MG, 1997. p. 57.

08. FUNDAÇÃO ONCOCENTRO DE SÃO PAULO. Manual de Enfermagem Oncológica. São Paulo: FOSP, s.d.

09. GATTAES, S. Toxicidade aguda dos quimioterápicos. In: LEITE, M.P.S. Câncer: diagnóstico e tratamento. São Paulo: Pert Plus, 1996. Parte 2. Cap. 13, p. 62-65.

10. GUTIÉRREZ, M.G.R. et al. Aprender, cuidar, pesquisar: a possível integração dessas práticas no curso de graduação. In: EXPÔ-ENF, São Paulo, 1995. Anais. São Paulo: UNIFESP/EPM, 1995. p. 66/Resumo.

11. HORTA, W.A. Processo de enfermagem. São Paulo: EPU/EDUSP, 1979.

12. Mc CLOSKEY, J.C.; BULECHEK, G.M. Nursing Interventions Classification (NIC), 2. ed. St. Louis: Mosby-Year Book, 1996. p. 53-68.

13. PEREIRA, L.L.; TAKAHSHI, R.T. Auditoria em enfermagem. In: KURCGANT, P. (coord.) Administração em Enfermagem. São Paulo: EPU, 1991. Cap. 17, p. 215-222. 